EPJ Web of Conferences 103, 04005 (2015)

DOI: $10.1051 /$ epjconf/ 201510304005

(C) Owned by the authors, published by EDP Sciences, 2015

\title{
Solitary Wave Generation from Constant Continuous Wave in Asymmetric Oppositely Directed Waveguide Coupler
}

\author{
E.V. Kazantseva ${ }^{1 \mathrm{a}}$ and A.I. Maimistov ${ }^{1,2}$ \\ ${ }^{1}$ National Research Nuclear University"MEPhl", Kashirskoye sh., 31, Moscow, 115409, Russia \\ ${ }^{2}$ Moscow Institute of Physics and Technology, Dolgoprudnyi, Institutskii lane, 9, Moscow region, 141700, Russia
}

\begin{abstract}
In a model which describes asymmetric oppositely directed nonlinear coupler it was observed in numerical simulations a phenomenon of solitary wave generation from the input constant continuous wave set at the entrance of a waveguide with negative refraction. The period of solitary wave formation decreases with increase of the continuum wave amplitude.
\end{abstract}

Keywords: metamaterials, solitary waves, oppositely directional coupler

Propagation and interaction of coupled waves in asymmetric oppositely directed waveguide coupler is described by the system of equations for normalized amplitudes $e_{1}$ and $e_{2}$ of electric field of the light wave [1,2]:

$$
i\left(\frac{\partial}{\partial \zeta}+\frac{\partial}{\partial \tau}\right) e_{1}+e_{2}+r\left|e_{1}\right|^{2} e_{1}=0, i\left(\frac{\partial}{\partial \zeta}-\frac{\partial}{\partial \tau}\right) e_{2}-e_{1}=0
$$
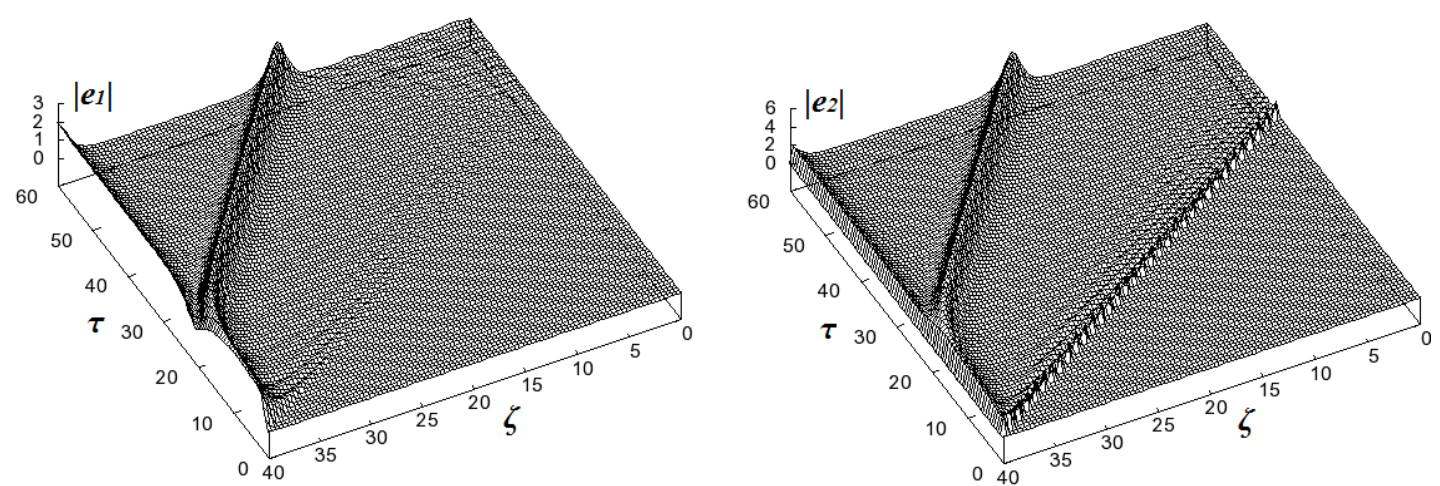

Figure 1. Formation of solitary wave from constant wave with amplitude equal to 2, which is set at the entrance of the waveguide with negative refraction in the asymmetric oppositely directed nonlinear coupler (in the end side of coupler at $\zeta=40$ ).

\footnotetext{
${ }^{\mathrm{a}}$ Corresponding author: elena.kazantseva@gmail.com
} 
In the numerical study of formation of solitary waves from the continuum the parameter of nonlinearity was set equal to $1(r=1)$. The amplitude of continuum wave at the input of waveguide with negative refraction was set to different values. At the amplitude of constant continuous wave equal to 2, the solitary wave appears from the continuum (see Fig.1).

Number of solitary waves grows with increase in amplitude of the constant wave. At the amplitude of constant wave (set in the entrance of the waveguide with negative refraction) equal to 2.5 , there are five solitary waves appeared in the asymmetric oppositely directed nonlinear coupler (Fig.2). At the amplitude equal to 4 there are eleven solitary waves appear (Fig.3).
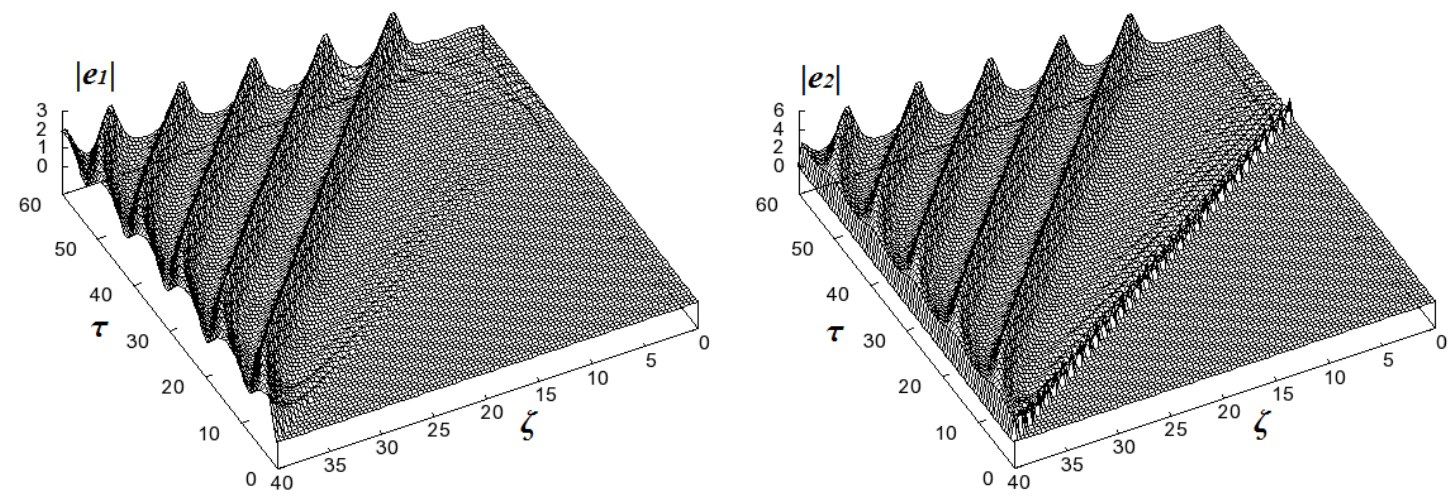

Figure 2. Formation of solitary waves from constant wave with amplitude equal to 2.5 , which is set at the entrance of the waveguide with negative refraction in the asymmetric oppositely directed nonlinear coupler (in the end side of the coupler at $\zeta=40$ ).
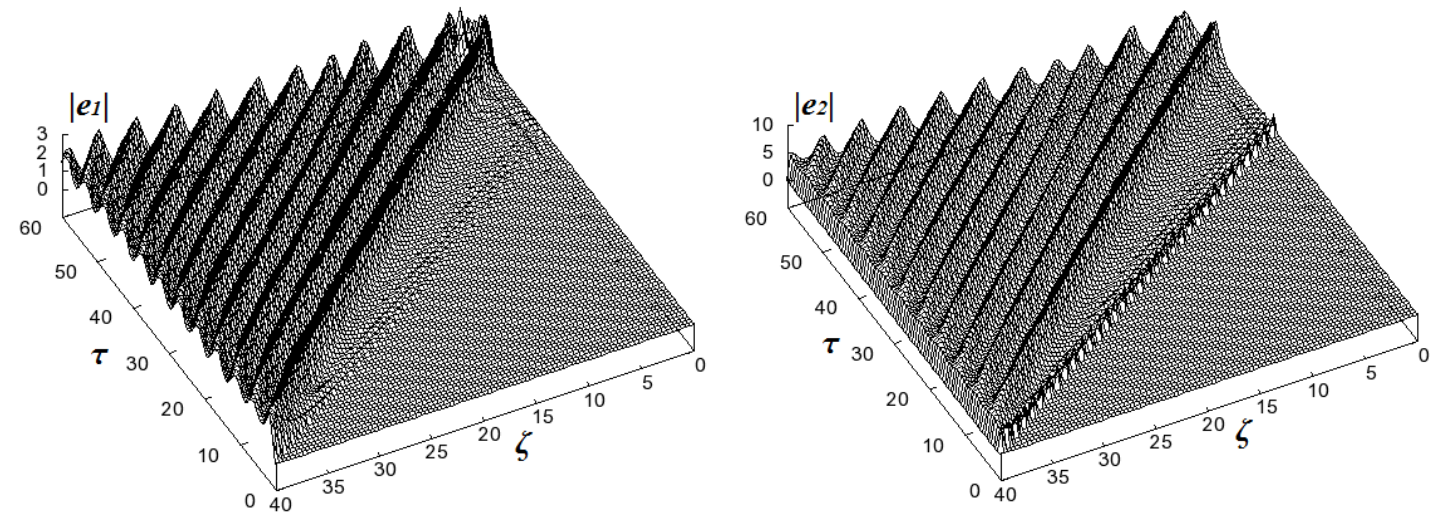

Figure 3. Formation of solitary waves from constant wave with amplitude equal to 4 , which is set at the entrance of the waveguide with negative refraction in the asymmetric oppositely directed nonlinear coupler (in the end side of the coupler at $\zeta=40$ ).

Summing up, a continuous wave of certain amplitude which is set at the entrance of the waveguide with negative refraction in the asymmetric oppositely directed nonlinear coupler could transform (due to nonlinear phase modulation) into a comb of solitary waves.

The research is supported by the Russian Foundation for Basic Research (Grant No. 15-02-02764).

\section{References}

1. E.V. Kazantseva, A.I. Maimistov, S.S. Ozhenko, Phys. Rev. A 80. 043833 (2009)

2. A.I. Maimistov, E.V. Kazantseva, S.S. Ozhenko, Bulletin of the Russian Academy of Sciences. Physics. 75, № 2. P. 165-167 (2011) 